\title{
Molecular Beam Studies of Primary Photochemical Processes
}

\author{
YUAN T. LEE
}

Materials and Molecular Research Division, Lawrence Berkeley Laboratory and Department of Chemistry, University of California, Berkeley, California 94720 USA

Application of the method of molecular beam photofragmentation translational spectroscopy to the investigation of primary photochemical processes of polyatomic molecules is described.

Examples will be given to illustrate how information concerning the energetics, dynamics and mechanism of dissociation processes can be obtained from the precise measurements of angular and velocity distributions of products in an experiment in which a well defined beam of molecules is crossed with a laser.

\section{INTRODUCTION}

There are many questions concerning the primary photochemical processes of simple polyatomic molecules which still remain to be answered. Some of the questions most frequently asked concern the mechanism and the relative importance of various dissociation channels, the nature of excited states involved, the energetics of product formation and the detailed dynamics of dissociation processes.

In many cases, the lack of a clear understanding of the primary photochemical processes is due to the experimental complication caused by either the collisions of excited molecules prior to dissociation or the secondary reactions of primary radical products. Sometimes, the difficulty arises because of the lack of a suitable experimental method to identify the primary polyatomic radical products which are highly internally excited. These highly excited polyatomic radicals often fragment extensively during the electron bombardment ionization and do not yield the parent ions in mass spectrometric 
detection. The lack of either sufficient spectroscopic information or distinct features in absorption or emission spectra also make it difficult to detect these excited radicals spectroscopically.

The complication caused by collisional processes can be eliminated by carrying out the experiment at a very low pressure or under collision free conditions of a molecular beam. The positive identification of primary radical products, which is also problematic in the elucidation of the mechanism of some bimolecular elementary chemical reactions, has also been shown to be possible, if high resolution molecular beam experiments are carried out using a mass spectrometric detector. From a careful comparison of angular and velocity distributions of products measured at various mass numbers which yield observable signals, and using kinematic relations together with the requirement of the conservation of energy and momentum, the mechanisms of a series of reactions involving oxygen atoms and unsaturated hydrocarbons have been successfully elucidated ${ }^{1,2}$ in spite of the severe fragmentation of products in the mass spectrometer.

The measurement of the velocity and angular distributions of photodissociation products in a molecular beam experiment has been referred to as the method of molecular beam photofragmentation translational spectroscopy. The usefulness of this method in the elucidation of dissociation dynamics and the characterization of repulsive electronic states has been fully demonstrated in pioneering research work carried out in the laboratories of Wilson ${ }^{3}$ and Bersohn. ${ }^{4}$ Recent advances in the development of high power lasers and improvements in the high intensity molecular beam source and the detection of scattered molecules makes this method an extremely useful and important tool for the investigation of primary photochemical processes. Much of the initial confusion concerning the dynamics of infrared multiphoton dissociation processes have been clarified by this method. ${ }^{5}$

In this article some examples and results of our recent molecular beam photofragmentation studies will be described.

\section{EXPERIMENTAL}

The experimental arrangement used in our molecular beam photofragmentation translational spectroscopy has been described pre- 
viously. ${ }^{6, /}$ Typically, a supersonic beam of molecules is formed in a nozzle-skimmer arrangement by expanding either a neat or a rare gas carried vapor through a $0.1 \mathrm{~mm}$ diameter nozzle. With a set of defining slits located on the walls of the two stage differential pumping chamber, the beam is defined to a $2^{\circ}$ angular divergence. The molecular beam is crossed by the output of a pulsed high power laser in the main chamber which is maintained at $1 \times 10^{-7}$ Torr.

Photofragments are detected by a triply differentially pumped mass spectrometer which rotates in the plane of the two beams about the crossing volume. Product time-of-flight spectra are recorded at various angles using a multichannel scaler triggered by the laser. The ionizer of the mass spectrometer is located, depending on the apparatus used, either $20.8 \mathrm{~cm}$ or $34.1 \mathrm{~cm}$ from the intersection region of the laser and the molecular beam. Because of the defining slits on the detector chambers, only photofragments originating in the crossing volume of approximately $3 \mathrm{~mm}$ on a side are obtained.

\section{EXAMPLES OF RECENT EXPERIMENTAL STUDIES}

\section{The dissociation dynamics of formaldehyde, $\mathrm{H}_{2} \mathrm{CO}^{8}$}

One of the intriguing questions in the photodissociation of formaldehyde is the nature of the dissociation process and the energy distribution of product molecules $\left(\mathrm{H}_{2}+\mathrm{CO}\right)$ when the formaldehyde is excited to an energy level near the zero point energy of the $S_{1}$ state. It became clear in recent years that the internal conversion to $S_{0}$ precedes the dissociation of formaldehyde, although some questions concerning the "mysterious intermediate state"

$A b$ initio quantum mechanical calculations have shown that the transition state of the dissociation of $\mathrm{H}_{2} \mathrm{CO}$ to $\mathrm{H}_{2}+\mathrm{CO}$ in the $S_{0}$ potential energy surface is coplanar with both hydrogen atoms lying on one side of the $\mathrm{CO}$ axis. ${ }^{10}$ The barrier for the dissociation was estimated to be about $80 \mathrm{kcal} / \mathrm{mol}^{11-13}$ which is almost identical to the excitation energy of the $S_{1}$ electronic state at the zero point. When the formaldehyde is excited near the zero point energy of $S_{1}$, almost all the energy deposited in the molecules, $80 \mathrm{kcal} / \mathrm{mol}$, will become potential energy at the transition state. This potential energy will be released in the production of $\mathrm{H}_{2}+\mathrm{CO}$ after passing through the potential energy barrier. 
The results of our recent molecular beam photofragmentation studies indicate that when formaldehyde is excited near the $S_{1}$ threshold, a large fraction of the available energy, $75 \%$ or $60 \mathrm{kcal} / \mathrm{mol}$, appears as translational energy of the $\mathrm{H}_{2}+\mathrm{CO}$ products, with a relatively narrow energy distribution. ${ }^{8}$ Since the $\mathrm{CO}$ produced was shown by Houston and Moore ${ }^{9}$ to be mostly in the ground vibrational state, even if most of the remaining energies were to be in the vibrational excitation of $\mathrm{H}_{2}$, the average vibrational quantum number of $\mathrm{H}_{2}$ should not exceed $v^{\prime}=2{ }^{8}$ The high translational energy in the dissociation products implies that most of the available energy is released repulsively between $\mathrm{H}_{2}$ and $\mathrm{CO}$ after the $\mathrm{H}_{2}$ bond is nearly fully developed and both $\mathrm{C}-\mathrm{H}$ bonds are broken.

The experimental observation of high translational energy release with a relatively narrow distribution in products has important consequences in understanding the nature of the "mysterious intermediate state" in the dissociation of formaldehyde. From the geometry of the transition state and the observed product translational energy distribution, the rotational distribution of $\mathrm{CO}$, which could be inverted, is expected to have an average quantum number of around $J^{\prime}=23$. This level of rotational excitation in CO contains only about $4 \mathrm{kcal} / \mathrm{mol}$ of energy, but the relatively high quantum number implies that the previous observation of the "delayed" production of $\mathrm{CO}$, which was attributed to the existence of the "mysterious intermediate state" might be in error. ${ }^{8}$ Because, the production of $\mathrm{CO}$ in that experiment was monitored by the absorption of $\mathrm{CO}$ in $J^{\prime}=10$ using a CW CO laser, the observed delay in the production of $\mathrm{CO}\left(J^{\prime}=10\right)$ was likely to be the rotational relaxation of $\mathrm{CO}$ which was produced "promptly" in higher rotational states rather than due to the existence of the "mysterious intermediate state."

The high rotational state distribution of the $\mathrm{CO}$ product concluded from the geometry of the transition state and the results of the measured translational energy distributions has been verified by Ho and $\mathrm{Smith}^{14}$ in a recent experiment in which $\mathrm{CO}$ rotational state distributions were measured directly by the laser induced fluorescence method.

\section{Dissociation mechanism of glyoxal, $\mathrm{H}_{2} \mathrm{C}_{2} \mathrm{O}_{2}{ }^{15}$}

Glyoxal is another molecule for which the vibronic energy levels are well resolved spectroscopically and are also fully characterized. 
Recent investigations of Loge and Parmenter ${ }^{16}$ have shown that glyoxal dissociates in the absence of collision to produce $\mathrm{H}_{2} \mathrm{CO}$ (formaldehyde), $\mathrm{CO}$ and $\mathrm{H}_{2}$ as products when it is excited to the threshold of the $S_{1}$ electronic state $(\sim 60 \mathrm{kcal} / \mathrm{mol})$. Although the formation of $\mathrm{H}_{2} \mathrm{CO}$ and $\mathrm{CO}$ is understood to be the result of the 1,2-hydrogen atom migration after the electronically excited molecules are internally converted to the highly vibrationally excited ground states molecules, the production of $\mathrm{H}_{2}$ is somewhat puzzling. Since the maximum possible internal excitation of $\mathrm{H}_{2} \mathrm{CO}$ formed after the excitation of glyoxal to near the $S_{1}$ threshold is only around $60 \mathrm{kcal} / \mathrm{mol}$, which is significantly less than the potential energy barrier $(\sim 80 \mathrm{kcal} / \mathrm{mol})^{11-13}$ of the dissociation of $\mathrm{H}_{2} \mathrm{CO}$, the secondary decomposition of vibrationally excited $\mathrm{H}_{2} \mathrm{CO}$ is not likely. There is also no known radical dissociation channel at this excitation energy which could eventually yield $\mathrm{H}_{2}$ as secondary products.

Extensive $a b$ initio calculations intended for the understanding of the decomposition of glyoxal by Osamura et al. ${ }^{17}$ have shown that a concerted reaction involving four-center elimination of $\mathrm{H}_{2}$ with a simultaneous rupture of the $\mathrm{C}-\mathrm{C}$ bond to form two $\mathrm{CO}$ molecules from a cis configuration of glyoxal has an unexpectedly low potential energy barrier of $55 \mathrm{kcal} / \mathrm{mol}$, which is comparable to that of 1,2 hydrogen atom migration forming $\mathrm{H}_{2} \mathrm{CO}$ and $\mathrm{CO}$. It suggests that the concerted reaction, $\mathrm{H}_{2} \mathrm{C}_{2} \mathrm{O}_{2} \rightarrow \mathrm{H}_{2}+2 \mathrm{CO}$, is probably as important as the formation of $\mathrm{H}_{2} \mathrm{CO}$ and $\mathrm{CO}$ in the photodissociation of glyoxal and $\mathrm{H}_{2}$ molecules are in fact one of the primary products.

In order to verify the existence and the importance of the concerted reaction forming $\mathrm{H}_{2}+2 \mathrm{CO}$, molecular beam photofragmentation experiments were carried out ${ }^{15}$ using a Nd:YAG laser pumped tunable dye laser system for excitation. The time-of-flight spectrum monitored at $m / e=30$ shows the existence of two peaks which correspond to two dynamically distinct channels forming $\mathrm{CH}_{2} \mathrm{O}+\mathrm{CO}$. From a consideration of the energies available for various possible channels and the measured translational energy distributions, it is concluded that the major peak with higher translational energy is due to the formation of $\mathrm{H}_{2} \mathrm{CO}$ (formaldehyde) and $\mathrm{CO}$, and the minor peak with lower translational energy is consistent with the formation of $\mathrm{HCOH}$ (hydroxymethylene) and $\mathrm{CO}$. The time-of-flight spectra obtained at $m / e=29\left(\mathrm{HCO}^{+}\right)$contain the same two peaks observed in the spectra of $m / e=30$. Apparently, $\mathrm{HCO}^{+}$ions are daughter ions of $m / e=30$ and not from the formation of the two HCO radicals 
from glyoxal. In fact, the excitation energy near the threshold of the $S_{1}$ state of glyoxal is not enough to break the $\mathrm{C}-\mathrm{C}$ bond to form two $\mathrm{HCO}$ radicals.

In the time-of-flight spectra of $m / e=28$, in addition to the two features which correspond to the formation of $\mathrm{H}_{2} \mathrm{CO}+\mathrm{CO}$ and $\mathrm{HCOH}+\mathrm{CO}$, there is an additional feature lying between those from $\mathrm{H}_{2} \mathrm{CO}+\mathrm{CO}$ and $\mathrm{HCOH}+\mathrm{CO}$. This new channel is an indication of the existence of a third $\mathrm{CO}$ producing channel which has nothing to do with the formation of the $\mathrm{CH}_{2} \mathrm{O}$ species. Although experimental difficulties prevented us from observing $\mathrm{H}_{2}$ molecules directly, there is no doubt that this third $\mathrm{CO}$ producing channel is indeed due to a concerted reaction forming $\mathrm{H}_{2}+2 \mathrm{CO}$. The branching ratios derived from the analysis of experimental results for the following dissociation channels

$$
\begin{aligned}
\mathrm{H}_{2} \mathrm{C}_{2} \mathrm{O}_{2}+h \nu & \rightarrow \mathrm{H}_{2} \mathrm{CO}+\mathrm{CO} \\
& \rightarrow \mathrm{H}_{2}+2 \mathrm{CO} \\
& \rightarrow \mathrm{HCOH}+\mathrm{CO}
\end{aligned}
$$

are $9: 3.5: 1$ for reactions $(1):(2):(3)$.

The formation of hydroxymethylene from glyoxal, which involves the 1,3 migration of the hydrogen atom from carbon atom to oxygen atom in the trans-configuration of glyoxal, was not suspected in previous experimental and theoretical studies, although there were some indications in earlier experimental studies of the possible existent of a radical channel which leads to the formation of some condensable products. ${ }^{16}$ Hydroxymethylene has not yet been observed in the gas phase experimental investigations. But, the stability of this radical has been reported in earlier ab initio calculations carried out by Goddard and Schaefer. ${ }^{10}$

\section{Energy splitting between singlet and triplet methylene, $\mathbf{C H}_{\mathbf{2}}{ }^{\mathbf{}}$}

Although photoelectron spectra obtained from the photodetachment of $\mathrm{CH}_{2}^{-}$gave the energy splitting between singlet methylene $\left[\mathrm{CH}_{2}(\mathrm{~S})\right]$ and triplet methylene $\left[\mathrm{CH}_{2}(\mathrm{~T})\right]$ to be $19 \mathrm{kcal} / \mathrm{mol},{ }^{18}$ most other experimental results and more rigorous $a b$ initio quantum mechanical calculations gave the value around 9 to $10 \mathrm{kcal} / \mathrm{mol} .{ }^{7}$ But, since the photodetachment experiment on $\mathrm{CH}_{2}^{-}$carried out by Lineberger and 
coworkers has been the only microscopic experiment making a direct observation on the quantities related to the energy splitting, the significant difference of as much as $10 \mathrm{kcal} / \mathrm{mol}$ on this splitting became one of the most interesting controversies in chemical physics in recent years.

In order to provide a second microscopic experiment using a different approach for the resolution of this controversy, the molecular beam photofragmentation translational spectroscopy on ketene, $\mathrm{CH}_{2} \mathrm{CO}$, was carried out recently. ${ }^{7}$

In the photofragmentation of an isolated molecule, because of the conservation of energy, the formation of products in the ground vibrational rotational state of a given electronic state should accompany the release of all the available energy in translational motion of products. In other words, the difference between the energy of exciting photons, $h \nu$, and the maximum translational energy released, $E_{m}^{\prime}$, in the dissociation, should provide the bond dissociation energy. Thus, if two laser frequencies, $\nu_{S}$ and $\nu_{T}$, were used for the dissociation of $\mathrm{CH}_{2} \mathrm{CO}$ to produce $\mathrm{CH}_{2}(S)+\mathrm{CO}$ and $\mathrm{CH}_{2}(T)+\mathrm{CO}$, respectively, and the maximum translational energies released for both cases, $E_{m}^{\prime}(S)$ and $E_{m}^{\prime}(T)$, are measured accurately in a molecular beam experiment, the energy splitting between the singlet and triplet methylene can be derived simply from the difference between the quantities $h \nu_{S}-E_{m}^{\prime}(S)$ and $h \nu_{T}-E_{m}^{\prime}(T)$.

This method avoids any complication which might be caused by the existence of exit potential energy barriers, since it does not depend on measurements of the thresholds for production of singlet and triplet methylene. However, it requires formation of some of the products in the ground vibrational-rotational state. The selection of $\mathrm{CH}_{2} \mathrm{CO}$ for this experiment has this consideration in mind. Because, the bond lengths of $\mathrm{CO}$ and $\mathrm{CH}$ in $\mathrm{CH}_{2} \mathrm{CO}$ are almost identical to that of $\mathrm{CO}$ and $\mathrm{CH}_{2}$, and the in plane bending vibrational motion of $\mathrm{CH}_{2}$ in $\mathrm{CH}_{2} \mathrm{CO}$ is directly coupled to the dissociation coordinate, even if a large fraction of excess energy appears as product internal excitation, some of the products are likely to be in the ground vibrational-rotational state.

Exciting $\mathrm{CH}_{2} \mathrm{CO}$ at $351 \mathrm{~nm}$, the only energetically allowed channel is $\mathrm{CH}_{2}(T)+\mathrm{CO}$. At $308 \mathrm{~nm}$, although the production of both singlet and triplet methylene are energetically allowed, almost all the products are the formation of $\mathrm{CH}_{2}(S)+\mathrm{CO}$. Our results of the 
measurements of product translational energy distributions show that $E_{m}^{\prime}(S)$ and $E_{m}^{\prime}(T)$ are 6.7 and $3.8 \mathrm{kcal} / \mathrm{mol}$ respectively, which, in turn, yields the singlet-triplet splitting of $8.5 \pm 0.8 \mathrm{kcal} / \mathrm{mol}{ }^{7}$ If the high value of $19 \mathrm{kcal} / \mathrm{mol}$ obtained in the photodetachment experiments is indeed incorrect, the electron affinity of $\mathrm{CH}_{2}$ obtained in the photodetachment experiment should also be increased by $\sim 10$ $\mathrm{kcal} / \mathrm{mol}$.

\section{CONCLUDING REMARKS}

The examples given above are only a small fraction of the photodissociation studies carried out by the molecular beam method. The works on the investigation of the dynamics of infrared multiphoton dissociation processes and the vibrational predissociation of hydrogen bonded molecules are some other examples.

It is also worth noting the important contributions made by the $a b$ initio quantum mechanical calculations in the understanding of all three examples given above. The ability to accurately calculate the geometry and the energy levels of transition states and radical species will become more and more important in all areas of chemistry in the future.

\section{Acknowledgement}

The examples given in this article are research works supported by the Office of Naval Research under the Contract Number N00014-75C-0671 and by the Director, Office of Energy Research, Office of Basic Energy Sciences Division of the US Department of Energy under Contract Number DE-AC03-76SF00098. Experiments on formaldehyde were carried out by Ho, Bamford and Buss in collaboration with Professor Moore's group. The work on glyoxal was carried out by Hepburn, Butler and Buss, and the energy splitting between singlet and triplet methylene was investigated by Hayden, Neumark, Shobatake and Sparks.

\section{References}

1. R. J. Buss, R. J. Baseman, G. He and Y. T. Lee, J. Photochem. 17, 389 (1981).

2. G. He, R. J. Baseman, R. Tse and Y. T. Lee, J. Phys. Chem. 86, 3547 (1982). 
3. G. E. Busch and K. R. Wilson, J. Chem. Phys. 56, 3626 (1972); G. E. Busch and K. R. Wilson, J. Chem. Phys. 57, 3638 (1972); G. E. Busch and K. R. Wilson, J. Chem. Phys. 56, 3655 (1972).

4. M. J. Dzvonik and S. C. Yang, Rev. Sci. Instrum. 45, 750 (1974); M. Kawasaki, S. J. Lee and R. J. Bersohn, J. Chem. Phys. 66, 2647 (1977).

5. E. R. Grant, M. J. Coggiola, P. A. Schulz, Aa. S. Sudbo, Y. R. Shen and Y. T. Lee, Chem. Phys. Lett. 52, 595 (1977); E. R. Grant, P. A. Schulz, Aa. S. Sudbo, M. J. Coggiola, Y. R. Shen and Y. T. Lee, in: Multiphoton Processes, eds. J. H. Eberly and P. Lambropoulos (J. Wiley and Sons, New York, 1978) p. 359.

6. R. K. Sparks, L. R. Carlson, K. Shobatake, M. L. Kowalczyk and Y. T. Lee, J. Chem. Phys. 72, 1401 (1980).

7. C. C. Hayden, D. M. Neumark, K. Shobatake, R. K. Sparks and Y. T. Lee, J. Chem. Phys. 76, 3607 (1980).

8. P. Ho, D. J. Bamford, R. J. Buss, Y. T. Lee and C. B. Moore, J. Chem. Phys. 76, 3630 (1982).

9. P. L. Houston and C. B. Moore, J. Chem. Phys. 65, 757 (1976).

10. J. D. Goddard and H. F. Schaefer, J. Chem. Phys. 70, 5117 (1979).

11. J. D. Goddard, Y. Yamaguchi and H. F. Schaefer, J. Chem. Phys. 75, 3459 (1981).

12. M. J. Frisch, R. Krishnan and J. A. Pople, J. Phys. Chem. 85, 1467 (1981).

13. G. F. Adams, G. D. Bent, R. J. Bartlett and G. D. Purvis, J. Chem. Phys. 75, 834 (1981).

14. P. Ho and A. Smith, Chem. Phys. Lett. in print (1982).

15. J. W. Hepburn, R. J. Buss, L. J. Butler and Y. T. Lee, submitted to J. Phys. Chem. (1982).

16. G. W. Loge and C. S. Parmenter, J. Phys. Chem. 85, 1653 (1981).

17. Y. Osamura, H. F. Schaefer, M. Dupuis and W. A. Lester, J. Cehm. Phys. 75, 5828 (1981).

18. P. C. Engelking, P. R. Corderman, J. J. Wendoloski, G. B. Ellison, S. V. O'Neil and W. C. Lineberger, J. Chem. Phys. 74, 5460 (1981). 